\title{
A New Approach for Measuring the Resilience of Transport Infrastructure Networks
}

\author{
Liang Wang $\mathbb{D},{ }^{1}$ Xiaolong Xue $\mathbb{D}^{2},{ }^{2}$ and Xun Zhou ${ }^{3}$ \\ ${ }^{1}$ School of Maritime Economics and Management, Dalian Maritime University, Dalian 116026, China \\ ${ }^{2}$ School of Management, Guangzhou University, Guangzhou 510006, China \\ ${ }^{3}$ Tippie College of Business, University of Iowa, Iowa City, Iowa 52242, USA \\ Correspondence should be addressed to Xiaolong Xue; xlxue@hit.edu.cn
}

Received 3 June 2020; Revised 23 July 2020; Accepted 29 July 2020; Published 17 August 2020

Guest Editor: Guangdong Wu

Copyright (C) 2020 Liang Wang et al. This is an open access article distributed under the Creative Commons Attribution License, which permits unrestricted use, distribution, and reproduction in any medium, provided the original work is properly cited.

Resilience is an important property of transport infrastructure networks. Resilient transport infrastructure networks can retain the performance in case of disturbances and quickly recover to the original performance level after the disturbances. This study proposes a new approach that can measure the resilience of transport infrastructure networks. The new approach gives a unified conceptual framework for measuring the resilience of transport infrastructure networks. A network simulation-based method is used to analyze the influential factors of transport infrastructure network resilience. A new measuring method is developed based on network diversity characteristic to quantitatively measure the system resilience and node resilience of transport infrastructure networks. China railway and air transport networks are selected as a case study to applicability of new approach. This new approach provides strong supports for academic and industrial fields to measure, analyze, enhance, and optimize the resilience of transport infrastructure networks.

\section{Introduction}

Compared to other types of critical infrastructure systems, transport infrastructure systems exhibit significant socialtechnology system characteristics [1]. As mega-projects and complex systems, transport infrastructure systems have a high degree of physical complexity and system complexity [2]. Complex systems are dynamic networks with self-organizing properties, which emerge from operating rules that govern the interactions of socioeconomic, ecological, climatic, and technical network components [3]. Many external disturbances and disasters have negative impacts on the performance of transport infrastructure network and can lead to the disruption or failure of transport infrastructure network [4-8]. Transport infrastructure network still faces significant challenges in enhancing resilience to various natural hazards and manmade threats.

A transport infrastructure network consists of different components, as railroad networks, highway networks, and airline networks. Due to the interactions between these components, disruptions or failure of a single component in a transport network might lead to cascading failures in other components. Further, an impact to a single node or link can result in important economic and physical damage at a citywide, regional, national, or even international scale [9]. Thus, transport infrastructure networks should be resilient and can maintain a certain degree of network performance when a disaster happens and can quickly recover to the original network performance level after the disaster $[10,11]$. The resilience of a transport infrastructure network reflects the ability of transport infrastructure system to handle disasters and is typically achieved through good designs of the network topological structure and characteristics [12].

In order to enhance the resilience of transport infrastructure networks, the first and essential step is to measure the resilience of transport infrastructure networks. State-ofthe-art measuring methods of system resilience have been summarized by Hosseini et al. and can be divided into qualitative assessment approaches and quantitative assessment approaches [13]. However, each of these measuring 
methods only focuses on a particular type of transport infrastructure system, such as road [14], railroad [15], or air transport [16]. None of these studies gave a unified conceptual framework for different types of transport infrastructure networks.

Other studies were proposed to measure the performance of transport infrastructure systems from the perspective of resilience. For example, Faturechi and Miller-Hooks suggested that the performance of transport infrastructure systems could be measured by using the TOSE model in disasters scenario [17]. Ouyang also reviewed the modeling and simulation of interdependent critical infrastructure systems [18]. However, these works do not measure the resilience directly based on the network topological structure properties and do not address our problem.

Considering the research gaps of existing measuring methods, it is necessary to propose a new approach that can measure the resilience of transport infrastructure networks. This new approach should give a unified conceptual framework for different categories of transport infrastructure networks. Under this unified conceptual framework, the influential factors of transport infrastructure network resilience will be identified and analyzed. A new method should also be developed to calculate and compare system resilience for different categories of transport infrastructure networks. In addition, the method should be able to measure node resilience and further identify the key nodes that have significant impacts on the resilience of the entire transport infrastructure network.

In this paper, the authors demonstrate a conceptual framework for measuring the resilience of transport infrastructure network with the above capabilities. The authors find through case studies that the resilience of transport infrastructure network is influenced by network nodes degree and distribution characteristic. A new measuring method is proposed based on network diversity.

This study begins with reviewing previous studies and explaining the research motivation. Then, a conceptual framework is demonstrated to measure resilience for different categories of transport infrastructure networks. A new measuring method is proposed for calculating the resilience of transport infrastructure networks from system level and nodes level. Finally, China railway and air transport networks resilience are measured at the system and node levels, and the matching of nodes importance and resilience is also tested.

\section{Literature Review and Research Motivation}

2.1. Measuring Methods on Transport Infrastructure Systems Resilience. The origins and meaning of system resilience are more diverse; thus it can be defined from different aspects. Holling defined resilience as the capacity to devise systems that could absorb and accommodate future events in whatever unexpected form they may take [19]. Allenby and Fink defined resilience as the capability of a system to maintain its functions and structure in the face of internal and external change and to degrade gracefully when it is a must [20]. MacKenzie and Barker defined resilience as the ability of a system to bounce back from a disruption [21]. These definitions reflect different characterizations of resilience. The discrepancies in the conceptual definitions of resilience arise from diverse epistemologies and methodology, and the measuring methods of resilience are also different in different research scenarios. Hosseini et al. reviewed existing measuring methods of resilience for different research areas; these measuring methods were divided into qualitative assessment approaches and quantitative assessment approaches [13]. The qualitative assessment approaches tended to assess the resilience of system without numerical descriptors. These qualitative assessment approaches either proposed conceptual frameworks that offered best practices to enhance resilience or offered expert assessments of different qualitative aspects of resilience $[13,22,23]$. The quantitative methods offered generic metrics to quantify resilience across applications and structural-based models to optimized system resilience $[13,14,18,24]$.

The first definition on resilience dates back to 1973 in the social and ecology domain [19]. Thus, most existing measuring methods were proposed from social and ecological perception. Transport infrastructure system exhibited significant social-technology system characteristics and presented networked external manifestation under the interactions of socioeconomic, ecological, climatic, and technical network components [1, 3, 25]. Most existing measuring methods could not be applied to transport infrastructure system. The authors summarized the measuring methods of transport infrastructure system resilience adopted from previous classic literatures studied by Henry and Ramirez-Marquez, Ouyang, Faturechi and MillerHooks, and Hosseini et al. [13, 14, 17, 18], and the classifications of reviewed papers are summarized in Table 1 along with their corresponding measuring methods on transport infrastructure system resilience.

Table 1 shows that transport infrastructure system resilience is usually measured by quantitative assessment methods in previous studies. Moreover, these measuring methods did not give a unified conceptual framework for different categories of transport infrastructure system. Most of quantitative assessment methods only applied to a single category of transport infrastructure system [14, 34]. Hosseini et al. divided these quantitative measuring methods into two subcategories: general resilience approaches and structural based modeling approaches [13].

General resilience approaches defined the resilience of transport infrastructure system from different dimensions and gave deterministic calculations from specific dimensions. Bruneau et al. defined four dimensions of resilience in the well-known resilience TOSE model in civil infrastructure, which also applied to transport infrastructure system [44]. Cox et al. assessed transportation security using direct static economic resilience (DSER), which was measuring from the likely maximum potential reduction given an external shock [26]. Henry and Emmanuel Ramirez-Marquez assumed that resilience was a time-dependent quantifiable metric that could be described by the ratio of recovery at time to loss suffered [14]. Enjalbert et al. 
TABLE 1: Measuring methods for transport infrastructure system resilience.

\begin{tabular}{|c|c|c|c|c|}
\hline Authors & Reference & Measuring method & Object studied in reference & Case location \\
\hline Cox et al. & {$[26]$} & Deterministic & Underground and buses & London \\
\hline Enjalbert et al. & [27] & Deterministic & Railway & French \\
\hline Woolley-Meza et al. & [28] & Deterministic & Air and cargo-ship transportation network & Global \\
\hline Henry and Emmanuel Ramirez-Marquez & {$[14]$} & Deterministic & Road network & Not specific \\
\hline Osei-Asamoah and Lownes & [29] & Deterministic & Highway and railway network & U.S \\
\hline Janić & {$[16]$} & Deterministic & Air transport network & U.S \\
\hline Pant et al. & {$[30]$} & Probabilistic & Waterway ports & U.S \\
\hline Vugrin et al. & {$[31]$} & Optimization & Transportation networks & Not specific \\
\hline Ouyang et al. & {$[32]$} & Optimization & Railway network & China \\
\hline Faturechi et al. & [33] & Optimization & Airport's runway & New York \\
\hline Faturechi and Miller-Hooks & {$[34]$} & Optimization & Roadway networks & Not specific \\
\hline Baroud et al. & {$[35]$} & Optimization & Waterway network & U.S \\
\hline Khaled et al. & {$[15]$} & Optimization & Railway network & U.S \\
\hline Wang and Ip & {$[36]$} & Simulation & Railway network & China \\
\hline Wuellner et al. & {$[37]$} & Simulation & Air transport network & U.S \\
\hline Chen and Miller-Hooks & {$[38]$} & Simulation & Transportation network & Not specific \\
\hline Ip and Wang & [39] & Simulation & Railway network & China \\
\hline Miller-Hooks et al. & {$[40]$} & Simulation & Railway network & U.S \\
\hline Cardillo et al. & {$[41]$} & Simulation & Air transport network & Europe \\
\hline Adjetey-Bahun et al. & {$[42]$} & Simulation & Railway network & Not specific \\
\hline Bhatia et al. & {$[43]$} & Simulation & Railway network & Indian \\
\hline
\end{tabular}

introduced local and global resilience assessment metrics that were used to measure transportation system resilience [27]. The above methods were deterministic performancebased approaches that did not consider uncertainty. Pant et al. proposed a probabilistic and stochastic measure of resilience which provided a more specific quantification of the value of resilience [30]. These general resilience approaches assessed the resilience of specific objects after disasters or hazards happened. Engineers could not assess the resilience status of transport infrastructure system using these general resilience approaches before disasters or hazards happened. In other words, these general resilience approaches cannot be used for disaster prevention. To overcome the shortages of general resilience approaches, scholars have presented many structural based modeling approaches for transport infrastructure system.

Compared with general resilience approaches, structural based modeling approaches could optimize the resilience of transport infrastructure system by analyzing the resilience status in different disasters and hazards scenarios. These optimization models can be used for both preevent prevention and postevent recovery. Scholars have designed various optimization models for transport infrastructure system resilience recently. Faturechi and Miller-Hooks applied three-stage stochastic program to maximize roadway travel time resilience and gave optimal plans for preevent mitigation and preparedness and postevent response [34]. Khaled et al. proposed a new optimization model and solution method to route freight rail traffic considering congestion and capacity at links and yards along with various operational constraints [15]. Vugrin et al. designed a bilevel optimization model for transport infrastructure network recovery and demonstrated a solution approach for this optimization model [31]. The above optimization models only statically assessed and analyzed the resilience of transport infrastructure system. With the increase of complexity in transport infrastructure system, simulation methods were used to assess transport infrastructure systems resilience from dynamic perspective. Adjetey-Bahun et al. used a time-dependent simulation model to measure the resilience indicators of a railway transportation system [42]. A set of disruptive events are modeled through simulation model with consequences of increase of travel time and reduction of train capacity. Miller-Hooks et al. measured the resilience of United States rail-based intermodal container network using L-shaped method and Monte Carlo simulation and found that resilient network had inherent ability to cope with disruption via its topological and operational attributes and potential actions that could be taken in the immediate aftermath of possible future disruptions [40]. Although the above structural based modeling approaches assess the resilience status of transport infrastructure system for preevent prevention, the real scenarios cannot be truly reflected in the process of prevention disasters.

Through reviewing previous studies on transport infrastructure system resilience, the authors found that transport infrastructure system resilience was usually measured by quantitative assessment methods. These quantitative assessment methods did not give a unified conceptual framework that could be used to analyze and compare the resilience of different transport infrastructure systems. Simultaneously, previous measuring methods were mainly optimization and simulation approaches that could not reflect the real disaster scenarios. Previous deterministic measuring methods were just used to assess the resilience of specific transport infrastructure systems after disasters or hazards happened. Thus, it is necessary to propose a new approach that can provide a unified conceptual framework for measuring the resilience of transport infrastructure systems. Under this unified conceptual framework, a new 
measuring method should also be developed to calculate and compare system resilience for different categories of transport infrastructure systems before disasters or hazards happened. All above discussions showed that there were still huge research gaps of existing measuring methods for transport infrastructure system resilience.

\subsection{Measuring Methods on Transport Infrastructure Networks} Resilience. Transport infrastructure systems have a high degree of physical complexity and system complexity and present significant network characteristics [2, 3]. Table 1 shows that most objects studied in references are transport infrastructure networks. Network-based approaches have become a new research paradigm for the resilience of transport infrastructure system [18, 45-47]. Transport infrastructure system can be described by networks, where nodes represent different network components and links manifest the relational connections and interactions among nodes. Previous studies have developed some network topology-based methods to analyze and measure the resilience of transport infrastructure network. Based on network topology-based methods, the value of network distribution $p_{k}$ is the probability that a randomly vertex is chosen in a node with network degree $k$ [48]. The resilience of network can be analyzed by different network topology-based methods, including the networks epidemic disease model [49], the breakdown of network under attacks [50], the networks boundaries $[51,52]$, and the cascading failures in network of networks [53]. In order to analyze the performance response of transport infrastructure networks under different hazards, scholars usually use the network simulation-based methods $[18,54]$. In previous studies related to network simulationbased methods, network performance can be measured by many metrics, such as the number of normal or failed network nodes [55], the path length among network nodes $[56,57]$, the loss ratio of network links [58], and the clusterrelated metrics [59].

As the research objects summarized in Table 1, most of objects studied in references are railway and air transport networks. Compared with general transport infrastructure system, railway and air transport networks resilience were usually measured and analyzed on a large scale. Railway and air transport networks nodes represented different cities and links manifested the transportation relationships among cities $[37,39]$. The impact to single city could result in important economic and physical damage on regional or even national scale $[16,29]$. Thus, railway and air transport networks were selected as case study in this study. The previous researches of railway and air transport networks resilience are reviewed in the following paragraphs.

The resilience measuring methods of railway and air transport networks in Table 1 are mainly optimization and simulation methods. The resilience of railway and air transport networks had been researched from different perspectives. Taking the railway network as an example, scholars have developed some network topology-based methods to analyze and measure the resilience of railway network. Ouyang et al. analyzed the resilience of China railway system through comparing their results with those from the real train flow model (RTFM) and found that the weighted shortest path model was more suitable for Chinese railway network resilience analysis [32]. Osei-Asamoah and Lownes evaluated structural resilience of Indiana interurban railroad network; the results showed that Indiana interurban railroad network could adapt to disruptions by optimizing network structures [29]. Bhatia et al. analyzed the impacts of the 2004 Indian Ocean Tsunami and the 2012 North Indian blackout on the resilience of Indian railways network and proposed network structure optimization method [43]. Network simulation-based methods were also used to measure and analyze the resilience of railway network in different hazards. Wang and IP proposed reliable independent pathbased resilience evaluation approach for railway networks in mainland China [36]. Ip and Wang evaluated and analyzed the resilience of railway network within the mainland China, where the results showed that nodes with high resilience are mainly located at the core of a railway network [39]. Miller-Hooks et al. measured the resilience of United States rail-based intermodal container network using L-shaped method and Monte Carlo simulation and found that resilient network had inherent ability to cope with disruption via its topological and operational attributes and potential actions [40].

Similarly, the measuring methods of air transport network were also mainly network topology-based methods and network simulation-based methods. Wuellner et al. analyzed the individual network topology structures of air transport networks in the USA, and the results showed that networks with dense interconnectivity were extremely resilient to both targeted and random attacks [37]. Woolley-Meza et al. analyzed the resilience of worldwide air-transportation network (WAN) and found the functional relationship between node characteristics and resilience [28]. Cardillo et al. studied the resilience of the European air transport network by using multiplex network formalism and found that the multiplicity strongly affects the resilience of the European air transport network [41]. Janić developed a methodology for estimating the resilience of an air transport network affected by a largescale disruptive event; the method is used to select real-life case under given conditions [16].

Through previous studies on the measuring methods of railway and air transport networks, the authors found that also there was no unified conceptual framework for measuring the resilience of railway and air transport networks. Most existing studies focused on analyzing network structure $[29,37,40]$ and its impacts on resilience of railway and air transport networks [28, 32, 39]. The resilience of railway and air transport networks could be enhanced by network topology-based methods proposed in previous studies $[16,41,43]$. Some studies referred to quantitative measures of network resilience [36], but the weight characteristics of transport infrastructure networks were not considered. Thus, railway and air transport networks had the same research gaps with general transport infrastructure networks on resilience measuring methods. 
To bridge the above research gaps, this study proposes a new approach that can measure the resilience of transport infrastructure networks. This new approach gives a unified conceptual framework for different categories of transport infrastructure networks. Under this unified conceptual framework, simulation method is used to analyze the influence factors of transport infrastructure network resilience. Considering the influence factors of transport infrastructure network resilience, a new measuring method is developed based on network diversity characteristic. This new measuring method can be used to quantitatively calculate and analyze the system resilience and nodes resilience of transport infrastructure networks. China railway and air transport networks are selected for case study to test the applicability of new approach. Scholars and engineers can use this new approach to measure the resilience of transport infrastructure networks under a unified conceptual framework. This new approach can also find key nodes that have significant impacts on the resilience of transport infrastructure networks. The key nodes should get more attentions in future disasters preprevention to avoid negative economic and physical impacts on a system, city-wide, regional, or even national or international scale. Thus, this new approach has great importance for enhancing the resilience of transport infrastructure networks.

\section{Approach Construction}

3.1. Conceptual Framework. Resilience Alliance designed a practitioner's workbook, which provided a generic framework for evaluating the resilience of social-ecological systems [60]. Hosseini et al. summarize the evaluating steps of the workbook: (i) defining and understanding the system under study, (ii) identifying the appropriate scale to evaluate resilience, (iii) identifying the system drivers and external and internal disturbance, (iv) identifying the key players in the system, including people and governance, (v) developing conceptual models for identifying necessary recovery activities, (vi) implementing the results of step $\mathrm{v}$ to inform policymaker, and (vii) incorporating the findings of the previous step [13]. This practitioner's workbook was designed from the perspective of social-ecological systems.

Compared with general social-ecological systems, transport infrastructure networks present physical complexity and system complexity $[2,61,62]$ and consist of different network components that interact with each other. Thus, the above practitioner's workbook is not applicable to measuring the resilience of transport infrastructure networks. The authors design a conceptual framework that measures the resilience of transport infrastructure networks from five steps: (i) defining and constructing transport infrastructure networks, (ii) identifying the levels to measure transport infrastructure networks resilience, which usually include nodes and system levels, (iii) analyzing the influence factors to transport infrastructure networks resilience, (iv) selecting appropriate measuring method for transport infrastructure networks resilience, and (v) calculating and analyzing the resilience of transport infrastructure networks. These five steps are specifically described in Figure 1.

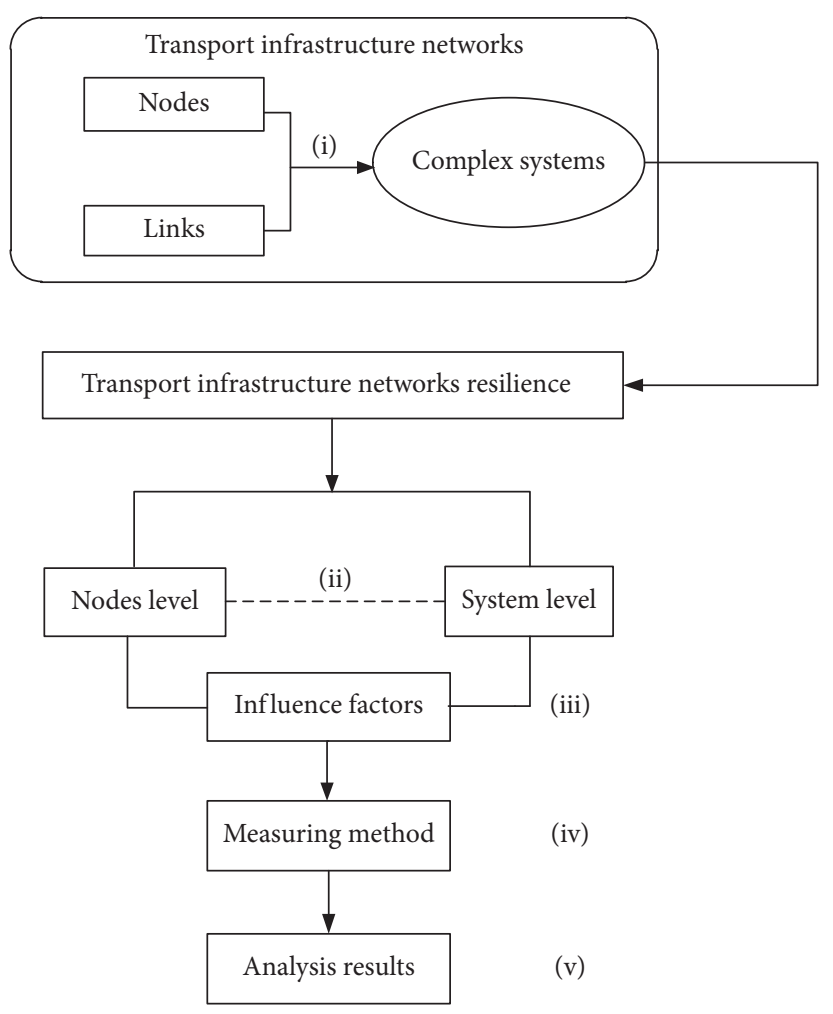

FIGURE 1: Assessment process of transport infrastructure networks resilience.

Figure 1 manifests that the first step of measuring transport infrastructure networks is defining and constructing transport infrastructure networks. Transport infrastructure networks consist of nodes and links, where nodes represent different network components and links manifest the relational connections and interactions among nodes. Next, transport infrastructure networks resilience is identified from nodes level or system level in step (ii), which depends on actual demands; system level resilience represents the comprehensive ability of transport infrastructure networks against disasters and hazards; this comprehensive ability can be qualitatively described and quantitatively measured; nodes level resilience represents the ability of single node against disasters and hazards in the context of transport infrastructure networks. Influence factors should be analyzed in step (iii), and influence factors identified will be used to design measuring method in step (iv). The resilience of transport infrastructure networks should be measured using appropriate method, and the measuring method is designed for specific transport infrastructure networks in step (iv). The resilience of transport infrastructure networks is measured and analyzed in step (v). Scholars and engineers can use this unified conceptual framework to measure the resilience of different transport infrastructure networks according to the above five steps.

3.2. Influence Factors Analysis. In this section, the authors analyze and identify the influence factors of network resilience, which is step (iii) of the conceptual framework 
proposed in Section 3.1. Network resilience can be influenced by different factors. Network system resilience is usually influenced by the network structure. The degree distribution of scale-free networks follows power law distribution, which means that scale-free networks show stronger resilience to random attacks caused by natural disasters, with weaker resilience to deliberate attacks against the central nodes, such as terrorist attacks [63].

Previous studies manifested that the failure of a node in a multinetwork system would not only reduce the performance of itself but also alter the network topology characteristics [64]. The network with appropriate connection patterns is highly resilient to random attack of nodes, and the hub nodes may be susceptible to an intentional attack [63]. The above process can be described as percolation on random graphs. Callaway et al. analyze percolation for the general case in which occupation probability is an arbitrary function of vertex degree [65]. The percolation is quantitatively described as the following equation:

$$
F_{0}(x)=\sum_{k=0}^{\infty} p_{k} q_{k} x^{k}
$$

In equation (1), $x$ is a specific network node, $k$ is the degree of network node $x, p_{k}$ is the probability that a randomly chosen vertex has degree $k, q_{k}$ is the probability that a vertex is occupied given that it has degree $k$, and $p_{k} q_{k}$ is the probability of having degree $k$ and being occupied. The authors simulate three scenarios of network percolation, which are shown in Figure 2.

Figure 2 presents three weighted networks, which are used to simulate different scenarios of network percolation. The red node is selected to analyze the influence factors of network node resilience. The numbers labelled along links are the link strengths between nodes; for example, the number 4 in scenario 1 represents that there are 4 links between red node and gray node. The authors use intentional attack strategy to simulate the scenario of network percolation. The node with the largest weight is first attacked. If there are some nodes with the greatest weight, each node has the same probability of being attacked. Node attack probability is quantitatively described as the following equation:

$$
p_{k}= \begin{cases}0, & \text { for } k \text { is not the node with the largest weight, } \\ 1, & \text { for } k \text { is the single node with the largest weight, } \\ \frac{1}{n}, & \text { for } k \text { is one of } n \text { nodes with the largest weight. }\end{cases}
$$

In equation (2), $p_{k}$ is node attack probability. When node $k$ is not the node with the largest weight, node $k$ will not be first attacked. When node $k$ is the single node with the largest weight, node $k$ will be first attacked. When node $k$ is one of $n$ nodes with the largest weight, node $k$ has the same probability of being first attacked as other nodes with the largest weight.
The authors use the standard degree to reflect the resilience performance of red node in Figure 2. The standard degree of red node after being attacked is calculated as the following equation:

$$
S_{D}=\frac{D_{\text {origin }}-D_{\text {loss }}}{D_{\text {origin }}} \times 100 .
$$

In equation (3), $S_{D}$ is the standard degree of node, $D_{\text {origin }}$ is the origin degree of node, and $D_{\text {loss }}$ is the loss degree of node. Three scenarios of network percolation are simulated by equations (2) and (3). The simulation result is shown in Figure 3.

The vertical axis and horizontal axis in Figure 3 are the change of node standard degree and attacks frequency, reflecting the response of standard degree in three network percolation scenarios. The simulation result shows that nodes resilience is influenced by network nodes degree and distribution characteristic. In scenario 1 , the degree of the red node is less than the degree of the red node in scenario 2. Compared to scenario 1 , the red node in scenario 2 has longer response time and higher resilience performance at different time, which means that the red node in scenario 2 exhibits higher resilience than that in scenario 1. Although the red node in scenario 3 has the same degree as that in scenario 2 , the red node in scenario 3 has higher resilience performance than that in scenario 2 at different time. The higher resilience is due to the uniform degree distribution of the red node in scenario 3 .

The above analysis results show that network nodes resilience is influenced by network nodes' degrees and distribution characteristic. The comparison result of scenario 1 and scenario 2 manifests that node with larger degree exhibits higher resilience. The comparison result of scenario 2 and scenario 3 manifests that node with uniform degree distribution characteristic exhibits higher resilience. Network nodes' degrees and distribution characteristic can be quantitatively measured by network diversity characteristic [66].

3.3. Network Resilience Measuring Method. In this section, the authors design the measuring method of transport infrastructure networks based on network diversity characteristic, which is step (iv) of conceptual framework proposed in Section 3.1. Transport infrastructure networks diversity refers to the network nodes and edges that contain different network status and functions and can maintain the original functions and states to the maximum extent in the event of disaster, which reflects the resilience of transport infrastructure networks [10]. The diversity of transport infrastructure networks is influenced by network nodes degree and distribution characteristic. The diversity characteristic reflects uncertainty, which can be calculated by entropy. Shannon entropy is the average information in an ensemble or event [67]; it is calculated as the following equation:

$$
H(X)=-\sum_{i=1}^{n} p\left(x_{i}\right) \log _{b} p\left(x_{i}\right) \text {. }
$$




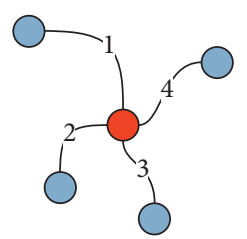

(a)

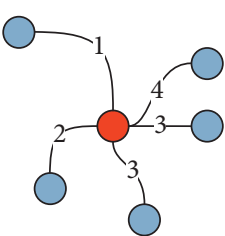

(b)

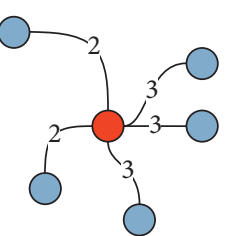

(c)

FIgURE 2: Three scenarios of network percolation. (a) Scenario 1. (b) Scenario 2. (c) Scenario 3.

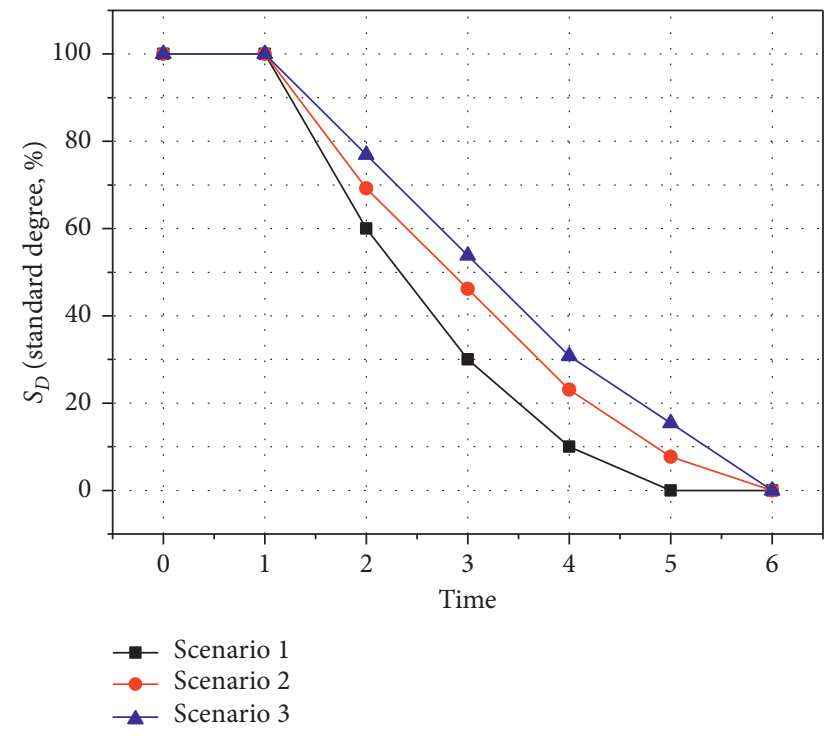

FIGURE 3: Response of standard degree in three network percolation scenarios.

In equation (4), $H(X)$ is the value of Shannon entropy. The content of Shannon entropy has to do with a particular outcome, $\log p(x)$, and entropy is based on a set of outcomes.

In this study, the Shannon entropy is used to quantify the diversity characteristic of transport infrastructure networks [66]. The resilience of transport infrastructure networks is calculated in system and node levels.

Node resilience of transport infrastructure network is calculated as the following equation:

$$
R_{i}=-\sum_{j=1}^{k} p_{i j} \log \left(p_{i j}\right) .
$$

In equation (5), $R_{i}$ is the resilience of node $i, k$ is the number of neighboring nodes connected with node $i, p_{i j}$ is the ratio of degree $D_{i j}$ between node $i$ and node $j$ against the total degree $D_{i}$ of links between node $i$ and its neighboring nodes, and $D_{i j}$ is the weight or number of links between node $i$ and node $j$.

$p_{i j}$ is calculated as the following equation:

$$
P_{i j}=\frac{D_{i j}}{\sum_{j=1}^{k} D_{i j}} .
$$

Transport infrastructure networks system resilience is calculated as the following equation:

$$
R=\sum_{i=1}^{n} w_{i} R_{i} .
$$

In equation (7), $R$ is the system resilience of transport infrastructure networks, and $n$ is the number of transport infrastructure networks nodes; $w_{i}$ is the weight of node $i . w_{i}$ is calculated as follows:

$$
w_{i}=\frac{D_{i}}{\sum_{i=1}^{n} D_{i}} .
$$

\section{Case Study}

4.1. China Railway and Air Transport Networks. In this section, the authors construct China railway and air transport networks, which is step (i) of conceptual framework proposed in Section 3.1. Compared with general transport infrastructure system, railway and air transport networks resilience were usually measured and analyzed on a large scale. Railway and air transport networks nodes represented different cities and links manifested the transportation relationships among cities $[37,39]$.

China railway and air transport networks are constructed in accordance with the following rules:

(1) Network nodes: Network nodes are prefecture-level cities that have railway or air transport access in China City Statistical Yearbook-2015 [68].

(2) Network links: If node $i$ can reach node $j$ directly by railway or air transport, there is a link between node $i$ and node $j$.

(3) Weighted network: The degree of link $i \longrightarrow j$ (denoted as $D_{i j}$ ) is defined as the number of different links from node $i$ to node $j$.

(4) Undirected network: Not all links of railway or air transport access between node $i$ and node $j$ appear in pairs. Thus, the degrees $D_{i j}$ and $D_{j i}$ between node $i$ and node $j$ are not completely symmetrical $\left(D_{i j} \approx D_{j i}\right)$. Here, each network is simplified as an undirected network based on the approximation $D_{i j} \longrightarrow\left(D_{i j}+D_{j i}\right) / 2$.

According to the above rules, this study uses the railway and civil aviation passenger transportation data of China railway customer service center [69] and Civil Aviation Administration of China [70] on November 15, 2015, to construct the China railway and air transport networks. 
China railway and air transport networks are depicted using Gephi software. Gephi software is a free tool for data analysts and scientists keen to explore and understand networks, and the main functions of Gephi software include real-time visualization, creating cartography, dynamic filtering, and layout and metrics analysis [71]. The specific construction steps are described as follows: Frist, the data of nodes and links are collected according to the rules proposed in Section 4.1. Then, nodes are mapped to specific cities, and the longitude and latitude of cities are identified. Finally, all data are imported into Gephi software by combining the plugins of GeoLayout and Maps of Countries layouts. China railway and air transport networks are shown in Figure 4.

The topological characteristics values of China railway and air transport networks are calculated, respectively: nodes number, degree, average degree, and connectivity degree. The calculation method refers to Woolley-Meza et al. [28]. Table 2 shows the topological characteristics of China railway and air transport networks.

Table 2 shows that the scale of China railway network is bigger than that of China air transport network. The nodes number and degree of China railway network are 2 and 7.8 times those of China air transport network. At the same time, the connection degree of China railway network is also better than that of China air transport network. The average degree and connectivity degree of China railway network are 4 and 2.1 times those of China air transport network.

4.2. Analysis Results. In this section, China railway and air transport networks are selected for case study to test the applicability of measuring method proposed in Section 3.3. China railway and air transport networks resilience are measured from system level and nodes level, which is step (ii) of conceptual framework proposed in Section 3.1. The measuring results of China railway and air transport networks resilience are analyzed, which is step v of conceptual framework proposed in Section 3.1.

4.2.1. System Resilience Analysis. The system resilience of China railway network and that of air transport networks are analyzed from the perspective of network structure characteristic. The system resilience is quantitatively calculated using the proposed measuring method. The degrees of nodes in China railway and air transport networks are divided into intervals of 100 and 10, respectively. Figure 5 shows the degree distribution of China railway and air transport networks.

Figure 5 shows the node degree frequency of China railway and air transport networks in different intervals. The curve is a power function fitting according to the scatter, which can be described as follows:

$$
F_{D}(k)=c k^{-\alpha}
$$

In equation (9), $F_{D}(k)$ is the number of nodes with degree $k ; c$ and $\alpha$ are the parameters values of power function. Table 3 gives the specific expression of the power function.
Figure 5 and Table 3 show that the degree distributions of China railway and air transport networks follow power law distribution, and China railway and air transport networks are scale-free networks. China railway and air transport networks show stronger resilience to random attacks caused by natural disasters and have weaker resilience to deliberate attacks against the central nodes, such as terrorist attacks [63]. According to the proposed measuring method of network resilience, the system resilience of China railway network and that of air transport networks are 1.7368 and 1.4699, which means that the system resilience of China railway network is stronger than that of China air transport network.

4.2.2. Node Resilience Analysis. Network node resilience reflects the ability of network node to maintain its original connection strength when it is attacked. Figure 6 shows the probability distribution of network node resilience in China railway and air transport networks based on the resilience measuring method proposed in this study.

Figure 6 shows that the probability distributions of network node resilience in China railway and air transport networks present different characteristics: (1) The nodes resilience of China railway network has significant differences in different intervals, and some network nodes show stronger resilience. The resilience of 82 network nodes is bigger than the system resilience of China railway network, which accounts for $30 \%$ of the total number of network nodes. (2) Compared to China railway network, the nodes resilience of China air transport network shows small differences in different intervals. Table 4 summarizes the top 10 cities that have the highest network node resilience in China railway and air transport networks.

Table 4 shows that the resilience of top 10 cities in China railway network is bigger than that of the top 10 cities in China air transport network. At the national level, 132 cities are the public nodes of China railway and air transport networks. The resilience of 123 cities in China railway network is bigger than their resilience in China air transport network. The top 10 cities with highest network node resilience in China railway network are important city nodes in China, such as municipalities (Beijing and Chongqing), regional centers (Chengdu, Wuhan, and Xi'an), and provincial capitals (Zhengzhou and Nanchang). The other three cities (Shangqiu, Xiangyang, and Hengshui) are also located near the boundaries between different provinces. The top 10 cities with highest network node resilience in China air transport network are all municipalities (Beijing, Shanghai, and Chongqing), regional centers (Xiamen and Shenzhen), and provincial capitals (Guangzhou, Kunming, Xi'an, Guiyang, and Haikou).

Most top 10 cities with highest network node resilience in China railway and air transport networks are municipalities, regional centers, and provincial capitals, which have played an important role in social development. To enhance the resilience of these municipalities, regional centers, and provincial capitals, the rescue capacity and recovery plan of cities are considered in the process of designating specific 


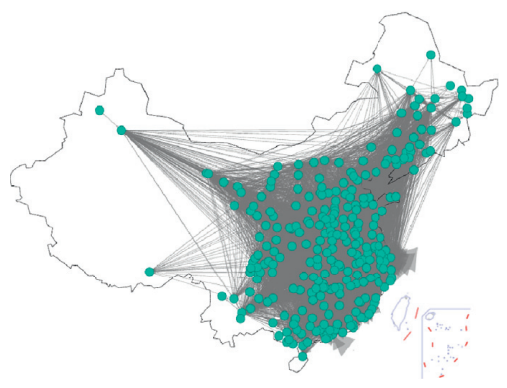

(a)

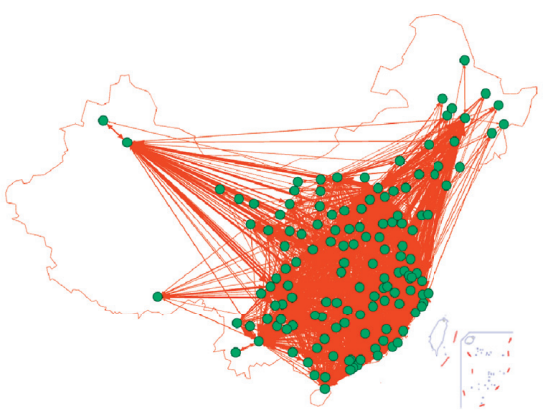

(b)

Figure 4: China railway and air transport networks. (a) Railway network. (b) Air transport network.

TABLE 2: Topological characteristics of China railway and air transport networks.

\begin{tabular}{lcccc}
\hline Topological characteristic & $N$ & $D$ & AD & \\
\hline Railway network & 273 & 136173 & 498.8 & 3.7 \\
Air transport network & 139 & 17424 & 125.4 & 1.8 \\
\hline
\end{tabular}

Note: $N$ is nodes number; $D$ is degree; $\mathrm{AD}=D / N$, which is average degree; $\sigma=2 D / N^{2}$, which is connectivity degree [28].

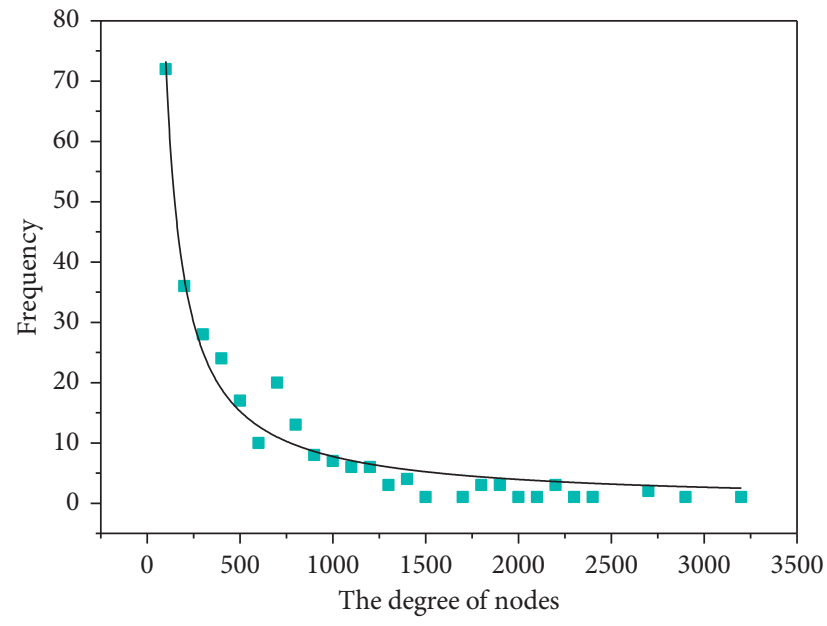

(a)

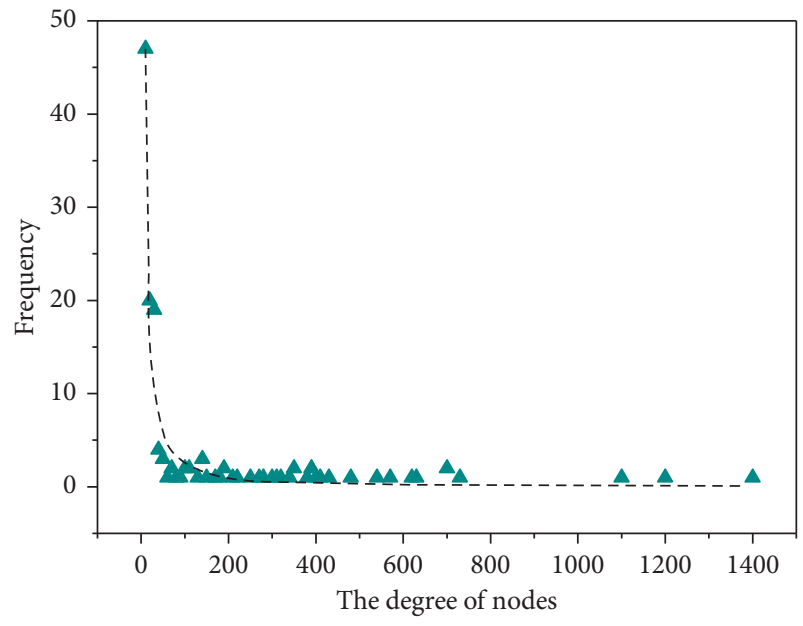

(b)

FIgURE 5: Node degree distribution of China railway and air transport networks. (a) Railway network. (b) Air transport network.

railway and air transport development plans. The nodes' degrees and diversity distribution characteristics of municipalities, regional centers, and provincial capitals are preferentially embedded into the specific rescue capacity and recovery plan of cities. Compared to other cities, more links are added between municipalities, regional centers, and provincial capitals to ensure the diversity distribution characteristic of network nodes degree.

Network nodes resilience of China railway and air transport networks shows nonuniform distribution characteristics in spatial regions. Most of cities with high node resilience are located in the eastern region. Figure 7 shows the imbalanced spatial distribution of network nodes resilience in China railway and air transport networks.

The straight line is Hu Huanyong Line in Figure 7, and the size of nodes represents the value of the node resilience.
Figure 7 shows that the node resilience of China railway and air transport networks presents great differences on both sides of Hu Huanyong Line. The number of network nodes on the eastern side of $\mathrm{Hu}$ Huanyong Line is much greater than that on the western side of Hu Huanyong Line, which is consistent with the imbalance in the spatial distribution of China population, economy, and industry. Overall, the network nodes resilience of China railway network in eastern region is higher than that of the western region. Table 5 summarizes the spatial distribution of network node resilience in China railway and air transport networks.

Table 5 quantitatively shows the imbalanced spatial distribution of network node resilience in China railway and air transport networks. The number of nodes and the average value of nodes resilience show great difference on both sides of Hu Huanyong Line. The number of network nodes in the 
TABLE 3: Power function parameters of China railway and air transport networks.

\begin{tabular}{lccr}
\hline Parameter & $c$ & $\mathrm{a}$ & $R^{2}$ \\
\hline Railway network & 6650.64 & 0.98 & 0.96 \\
Air transport network & 977.89 & 1.31 & 0.95 \\
\hline
\end{tabular}

Note: $R^{2}$ is the goodness of fit to power function.

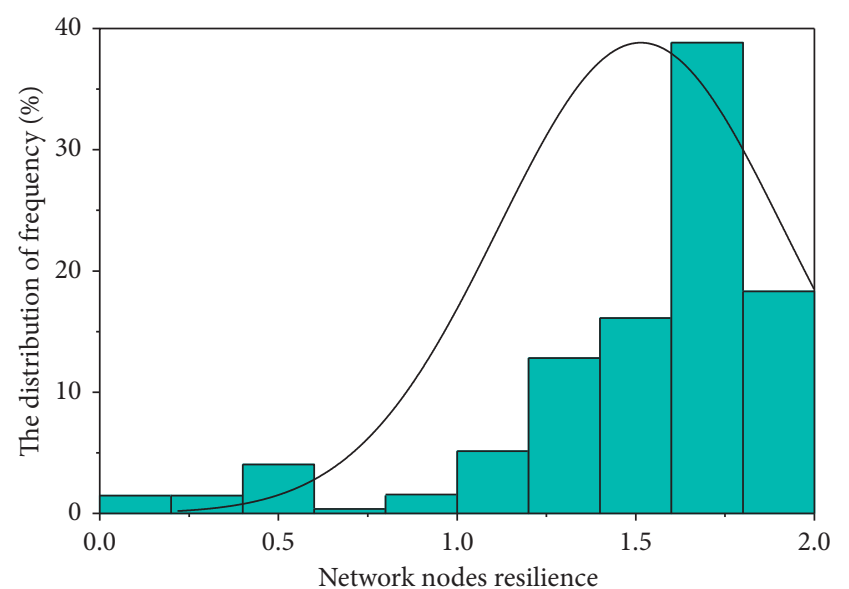

(a)

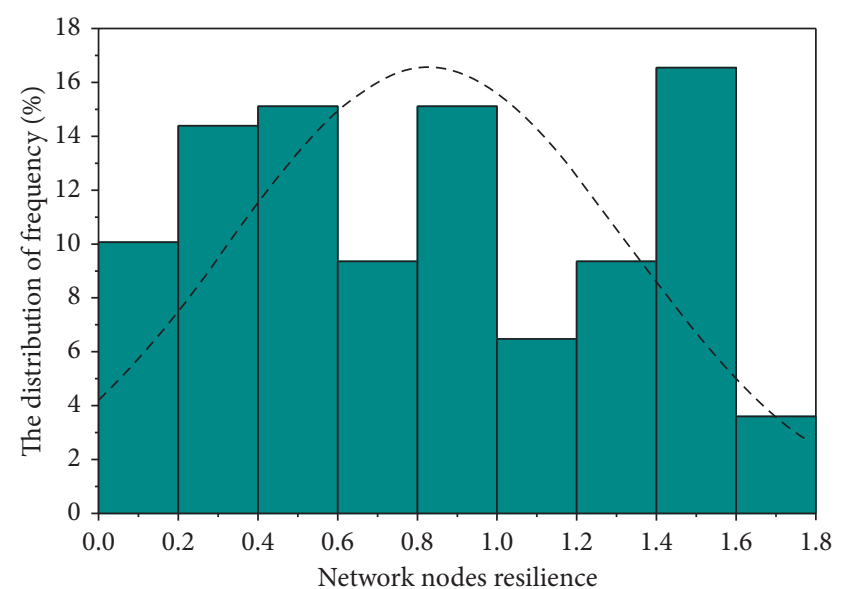

(b)

FIgURE 6: Node resilience distribution of China railway and air transport networks. (a) Railway network. (b) Air transport network.

eastern region accounts for $82.8 \%$ of the total number of network nodes in China railway network, and the average value of nodes resilience in eastern region is $1.1 \%$ higher than the western region. The number of network nodes in the eastern region accounts for $76.3 \%$ of the total number of network nodes in China air transport network, and the average value of nodes resilience in eastern region is $6.2 \%$ higher than that in the western region.

4.2.3. Matching of Nodes Importance and Resilience. The importance of network nodes can be described by different ways. The node degree reflects the direct influence of a node on the other nodes of the network; it has the advantages of simple intuition and low complexity [72]. This study uses node degree to describe the importance of nodes and analyzes its matching with node resilience. Figure 8 shows the basic principles of matching analysis on node importance and resilience.

According to the matching principles of node importance and resilience from low to high, Figure 8 is divided into four quadrants: (1) In quadrant I, node importance and resilience are both low. (2) In quadrant II, node importance and resilience are both high. (3) In quadrant III, node importance is low, and node resilience is high. (4) In quadrant IV, node importance is high, and node resilience is low. In this study, nodes degree and resilience of China railway and air transport networks are logarithmically normalized respectively. According to the matching principles given in Figure 8, Figure 9 shows the matching of nodes importance and resilience in China railway and air transport networks.
Figure 9 shows that there is a positive correlation between the nodes' importance and resilience of China railway and air transport networks. The node resilience increases with the increase of node importance, which means that nodes' importance and resilience are better matched in China railway and air transport networks. According to the matching principles given in Figure 8, nodes importance in quadrant III is lower than the average value; nodes resilience in quadrant III is higher than the average value. There is redundancy in the nodes resilience of quadrant III. Nodes importance in quadrant IV is higher than the average value; nodes resilience in quadrant IV is lower than the average value. Nodes resilience needs to be improved in quadrant IV. Nodes in quadrant III and quadrant IV present the poor matching of nodes importance and resilience. Table 6 gives the cities with the poor matching of nodes importance and resilience in quadrant III and quadrant IV.

Table 6 shows that there are 52 cities falling in quadrant III and quadrant IV in China railway and air transport networks. In addition to Dalian, the other 51 cities do not include important cities such as municipalities, regional centers, and provincial capitals, again showing the good matching of nodes importance and resilience in China railway and air transport networks. There are 7 common cities in China railway and air transport networks which fall in quadrant III; these 7 cities are Datong, Foshan, Huai'an, Nantong, Taizhou, Yan'an, and Yancheng. There are improvement rooms for the nodes importance of these 7 cities at the existing level of nodes resilience. There are 32 cities in China railway network which fall in quadrant III, and 10 cities fall in quadrant IV. The numbers account for $11.7 \%$ and $3.7 \%$ of the total number of cities. There are 16 cities in 
TABLE 4: Resilience of top 10 cities in China railway and air transport networks.

\begin{tabular}{lcccc}
\hline \multirow{2}{*}{ Ranking } & \multicolumn{2}{c}{ Railway network } & \multicolumn{2}{c}{ Air transport network } \\
& City & Resilience value & City & 1.7482 \\
1 & Beijing & 1.9992 & Beijing & 1.6767 \\
3 & Chengdu & 1.9946 & Kuangzhou & 1.6365 \\
3 & Chongqing & 1.9665 & Xi'an & 1.6325 \\
5 & Shangqiu & 1.9586 & Shanghai & 1.6264 \\
6 & Zhengzhou & 1.9515 & Chongqing & 1.5834 \\
7 & Wuhan & Guiyang & 1.5801 \\
8 & Xi'an & 1.9498 & Xiamen & 1.5650 \\
9 & Xiangyang & 1.9425 & Haikou & 1.5516 \\
10 & Nanchang & 1.9353 & Shenzhen & 1.5374 \\
\hline
\end{tabular}

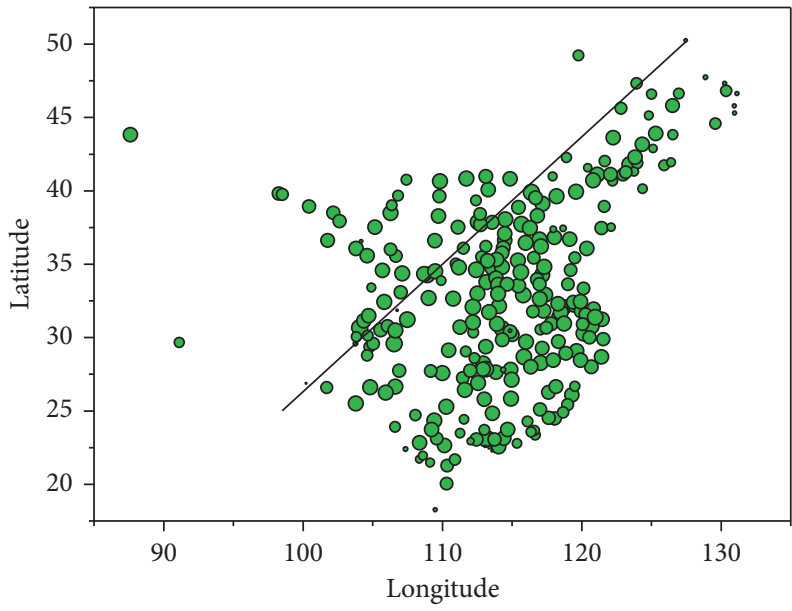

(a)

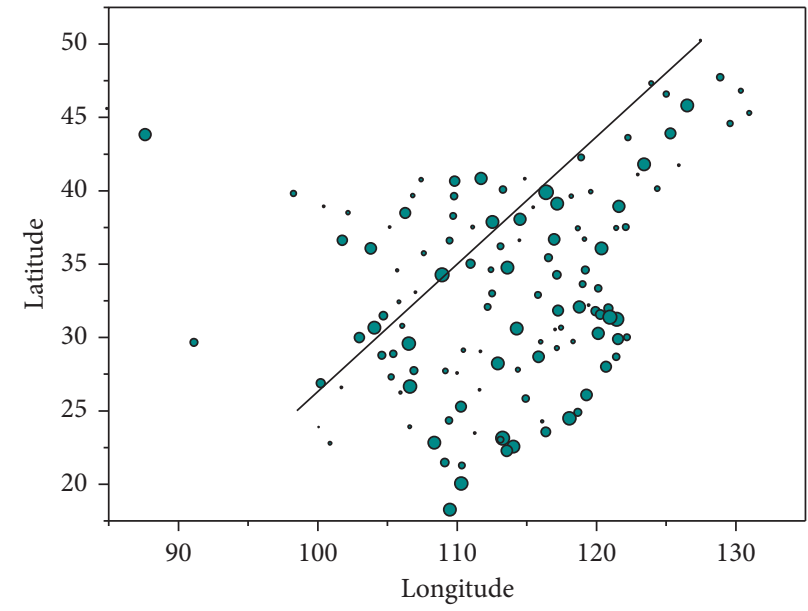

(b)

FIGURE 7: Spatial distribution of resilience in China railway and air transport networks. (a) Railway network. (b) Air transport network.

TABLE 5: Spatial distribution characteristics of nodes resilience.

\begin{tabular}{lccc}
\hline Network category & Region & Number of nodes & The average value of nodes resilience \\
\hline \multirow{2}{*}{ Railway network } & Eastern region & 226 & 1.7386 \\
& Western region & 46 & 1.7202 \\
\multirow{2}{*}{ Air transport network } & Eastern region & 106 & 1.4859 \\
& Western region & 32 & 1.3998 \\
\hline
\end{tabular}

China air transport network which fall in quadrant III, and 2 cities fall in quadrant IV. The numbers account for $11.5 \%$ and $1.4 \%$ of the total number of cities. From the perspectives of absolute and relative nodes number, the matching of nodes importance and resilience in China air transport network is better than that in China railway networks.

\section{Discussions}

Transport infrastructure systems present significant network characteristics and can be described by networks, where nodes represent different network components and links manifest the interactions among nodes [2, 3]. Thus, network-based approaches have become a new research paradigm for the resilience of transport infrastructure system [18]. In order to enhance the resilience of transport infrastructure networks, it is important to know how to measure the resilience of transport infrastructure networks. Although previous studies have proposed some network topology-based methods and network simulation-based methods to measure the resilience of transport infrastructure networks, there are still huge research gaps of existing measuring methods for transport infrastructure networks resilience. Existing measuring methods do not provide unified conceptual framework for measuring the resilience of transport infrastructure networks. Scholars and engineers do not know how to use a specific measuring method to assess the resilience of other transport infrastructure 


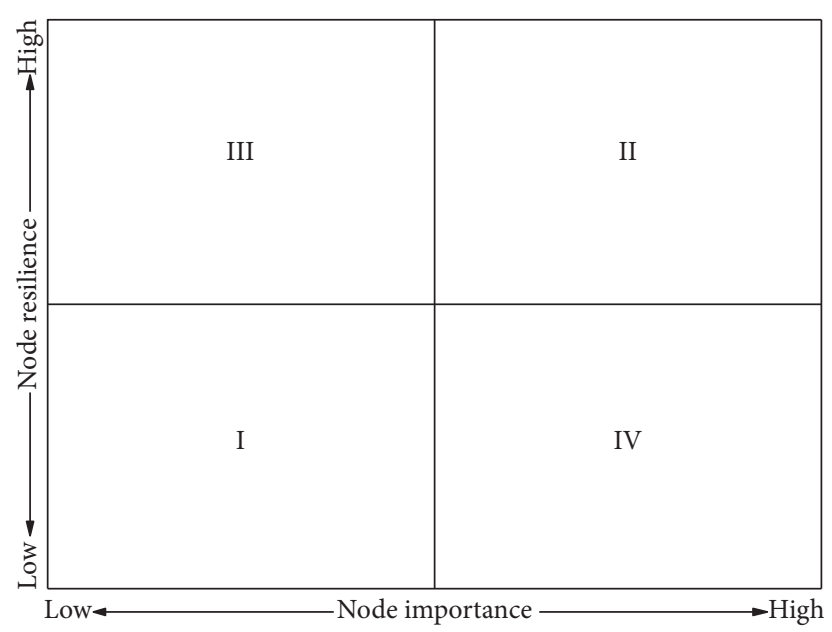

FIGURE 8: Matching principles of node importance and resilience.

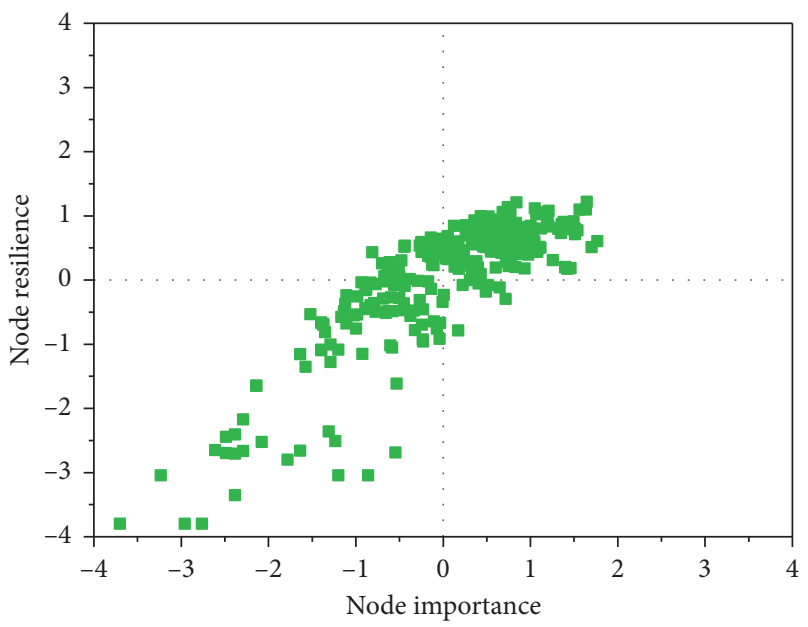

(a)

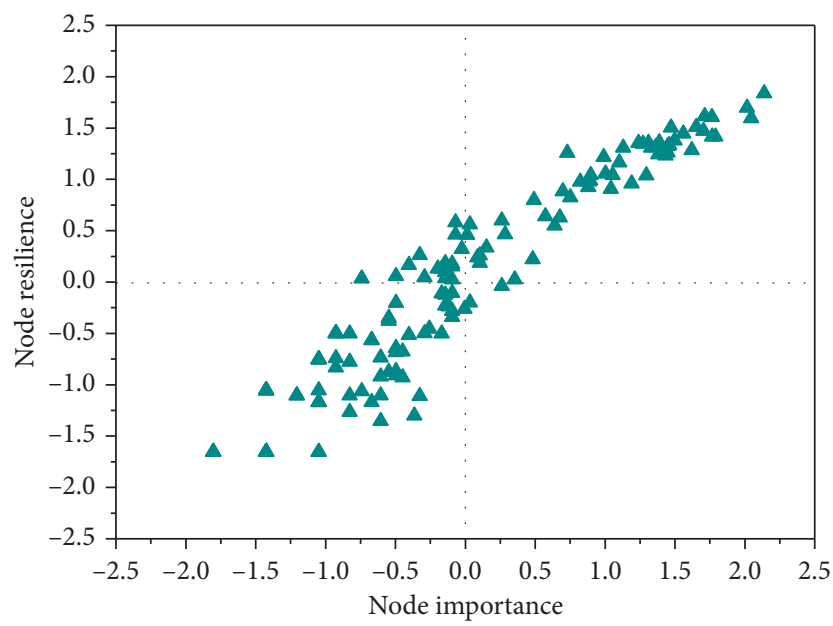

(b)

FIGURE 9: Matching of nodes importance and resilience. (a) Railway network. (b) Air transport network.

TABLE 6: Cities with poor matching in China railway and air transport networks.

\begin{tabular}{|c|c|c|}
\hline $\begin{array}{l}\text { Network } \\
\text { category }\end{array}$ & Quadrant III & Quadrant IV \\
\hline $\begin{array}{l}\text { Railway } \\
\text { network }\end{array}$ & $\begin{array}{c}\text { Jingmen, Guangyuan, Yinchuan, Yan'an, Zhangjiajie, Taizhou, Lishui, } \\
\text { Datong, Taizhou, Tongliao, Zhongwei, Yulin, Foshan, Jiaozuo, } \\
\text { Zhoukou, Suining, Luliang, Jinzhong, Yangzhou, Jingdezhen, Huaibei, } \\
\text { Ordos, Longyan, Yantai, Zunyi, Nantong, Hanzhong, Chizhou, } \\
\text { Zhanjiang, Yancheng, Huai'an, Tongren }\end{array}$ & $\begin{array}{l}\text { Shanwei, Quanzhou, Jiuquan, Dalian, Putian, } \\
\text { Huzhou, Lu'an, Guang, Anshan, Liaoyang }\end{array}$ \\
\hline $\begin{array}{l}\text { Air transport } \\
\text { network }\end{array}$ & $\begin{array}{l}\text { Nantong, Mianyang, Lianyungang, Jining, Luzhou, Ganzhou, Taizhou, } \\
\text { Datong, Liuzhou, Yichun, Yancheng, Yan'an, Foshan, Weihai, Huai'an, } \\
\text { Xiangyang }\end{array}$ & Chifeng, Zhanjiang \\
\hline
\end{tabular}

networks. Most of existing measuring methods are network topology-based methods and network simulation-based methods. The resilience of transport infrastructure network can be optimized by different network topology-based methods. Network simulation-based methods are used to analyze the performance response of transport infrastructure networks resilience under different hazards scenarios. Neither network topology-based methods nor network simulation-based methods can quantitatively measure the resilience of transport infrastructure networks. 
Simultaneously, previous measuring methods cannot reflect the real disaster scenarios and are just used to assess the resilience of transport infrastructure networks after disasters or hazards happened.

This study proposes a new approach that gives a unified conceptual framework for measuring the resilience of transport infrastructure networks. This unified conceptual framework measures the resilience of transport infrastructure networks from five steps. Scholars and engineers can measure the resilience of different transport infrastructure networks according to this unified conceptual framework. Thus, the resilience measuring results of different transport infrastructure networks can be compared with each other, such as railway network and air transport network in this study. Under the unified conceptual framework, network simulation-based method is used to analyze the influence factors of transport infrastructure network resilience. Thus, influence factors identified in this study can influence the resilience of transport infrastructure networks in different hazards scenarios, which reflects a dynamic perspective. The analysis results manifest that the resilience of transport infrastructure networks is influenced by network structure characteristic and degrees distribution characteristic. Considering the above influence factors, a new measuring method is developed based on network diversity characteristic. This new measuring method can quantitatively measure and analyze the system resilience and nodes resilience of transport infrastructure networks. The conceptual framework and measuring method proposed in this study bridge the gaps of existing research on transport infrastructure networks resilience. This new approach provides strong supports for academic and industrial fields to measure, analyze, enhance, and optimize the resilience of transport infrastructure networks.

\section{Conclusions}

Resilience is an important property of transport infrastructure networks. How to measure the resilience of transport infrastructure networks has become an important research topic. The research motivation is elaborated by reviewing previous researches on the measuring methods of transport infrastructure networks resilience. In order to bridge the gaps of existing researches, this study proposes a new approach that gives a unified conceptual framework for measuring the resilience of transport infrastructure networks. Network simulation-based method is used to analyze the influence factors of transport infrastructure network resilience. A new quantitative measuring method is developed based on network diversity characteristic. China railway network resilience and air transport network resilience are selected as a case study to test applicability of new approach.

Compared with previous measuring methods, the new approach proposed in this study is more suitable for measuring the resilience of transport infrastructure networks. Scholars and engineers can use this new approach to measure the resilience of transport infrastructure networks under a unified conceptual framework, which means that the resilience measuring results of different transport infrastructure networks can be compared with each other. The new measuring method can quantitatively measure the resilience of transport infrastructure networks and find the key nodes with significant impacts on the resilience of transport infrastructure network. The measuring results of resilience provide supports for future transport infrastructure networks operation and management.

This study is a preliminary study on the measuring methods of transport infrastructure networks. Systems can achieve resilient status through three mechanisms: persistence, transition, and transformation [73, 74]. This study assumes that transport infrastructure networks achieve resilient status through persistence mechanisms. Additional studies are required to propose the measuring methods of transport infrastructure networks from transition and transformation mechanisms. In addition, the approach proposed in this study is only suitable for measuring the resilience of single-layer network. Advanced measuring methods should be designed for multilayer compound networks, and the interdependency effect of multilayer compound networks should be considered in future research $[18,75,76]$.

\section{Data Availability}

The data used to support the findings of this study are available from the corresponding author upon request.

\section{Conflicts of Interest}

The authors declare that they have no conflicts of interest.

\section{Acknowledgments}

This research was funded by the National Social Science Fund of China (no. 18ZDA043). The work described in this paper was also supported by the National Natural Science Foundation of China (NSFC) (nos. 71671053, 71841024, 71771067 , and 71390522), the National Key R\&D Program of China (nos. 2016YFC0701800 and 2016YFC0701808), the Department of Science and Technology of Guangdong Province (no. 2019B101001019), and the Fundamental Research Funds for the Central Universities (no. 3132020224).

\section{References}

[1] T. P. Dunn and J. Sussman, "Simulation of impact of strategy development frameworks on performance of transportation infrastructure," Transportation Research Record: Journal of the Transportation Research Board, vol. 2255, no. 1, pp. 165-176, 2011.

[2] M. Lehtonen, "Evaluating megaprojects: from the "iron triangle" to network mapping," Evaluation, vol. 20, no. 3, pp. $278-295,2014$.

[3] E. Porse and J. Lund, "Network structure, complexity, and adaptation in water resource systems," Civil Engineering and Environmental Systems, vol. 32, no. 1-2, pp. 143-156, 2015.

[4] P. Bocchini, D. M. Frangopol, T. Ummenhofer, and T. Zinke, "Resilience and sustainability of civil infrastructure: toward a 
unified approach," Journal of Infrastructure Systems, vol. 20, no. 2, Article ID 04014004, 2013.

[5] A. Alipour and B. Shafei, "Assessment of postearthquake losses in a network of aging bridges," Journal of Infrastructure Systems, vol. 22, no. 2, Article ID 04015023, 2016.

[6] X. Zhang and E. Miller-Hooks, "Scheduling short-term recovery activities to maximize transportation network resilience," Journal of Computing in Civil Engineering, vol. 29, no. 6, Article ID 04014087, 2015.

[7] W. Wisetjindawat, A. Kermanshah, S. Derrible, and M. Fujita, "Stochastic modeling of road system performance during multihazard events: flash floods and earthquakes," Journal of Infrastructure Systems, vol. 23, no. 4, Article ID 04017031, 2017.

[8] L. Wang, X. Xue, Z. Wang, and L. Zhang, "A unified assessment approach for urban infrastructure sustainability and resilience," Advances in Civil Engineering, vol. 2018, Article ID 2073968, 19 pages, 2018.

[9] E. C. Portante, J. A. Kavicky, B. A. Craig, L. E. Talaber, and S. M. Folga, "Modeling electric power and natural gas system interdependencies," Journal of Infrastructure Systems, vol. 23, no. 4, Article ID 04017035, 2017.

[10] C. Mason and E. Strankalis, "Network resilience: a new way to target maintenance spending," Proceedings of the Institution of Civil Engineers-Civil Engineering, vol. 164, no. 4, p. 149, 2011.

[11] B. Genge and C. Siaterlis, "Analysis of the effects of distributed denial-of-service attacks on MPLS networks," International Journal of Critical Infrastructure Protection, vol. 6, no. 2, pp. 87-95, 2013.

[12] X. Zhang, E. Miller-Hooks, and K. Denny, "Assessing the role of network topology in transportation network resilience," Journal of Transport Geography, vol. 46, pp. 35-45, 2015.

[13] S. Hosseini, K. Barker, and J. E. Ramirez-Marquez, "A review of definitions and measures of system resilience," Reliability Engineering \& System Safety, vol. 145, pp. 47-61, 2016.

[14] D. Henry and J. Emmanuel Ramirez-Marquez, "Generic metrics and quantitative approaches for system resilience as a function of time," Reliability Engineering \& System Safety, vol. 99, pp. 114-122, 2012.

[15] A. A. Khaled, M. Jin, D. B. Clarke, and M. A. Hoque, "Train design and routing optimization for evaluating criticality of freight railroad infrastructures," Transportation Research Part B: Methodological, vol. 71, pp. 71-84, 2015.

[16] M. Janić, "Modelling the resilience, friability and costs of an air transport network affected by a large-scale disruptive event," Transportation Research Part A: Policy and Practice, vol. 71, pp. 1-16, 2015.

[17] R. Faturechi and E. Miller-Hooks, "Measuring the performance of transportation infrastructure systems in disasters: a comprehensive review," Journal of Infrastructure Systems, vol. 21, no. 1, Article ID 04014025, 2015.

[18] M. Ouyang, "Review on modeling and simulation of interdependent critical infrastructure systems," Reliability Engineering \& System Safety, vol. 121, pp. 43-60, 2014.

[19] C. S. Holling, "Resilience and stability of ecological systems," Annual Review of Ecology and Systematics, vol. 4, no. 1, pp. 1-23, 1973.

[20] B. Allenby and J. Fink, "Toward inherently secure and resilient societies,” Science, vol. 309, no. 5737, pp. 1034-1036, 2005.

[21] C. A. MacKenzie and K. Barker, "Empirical data and regression analysis for estimation of infrastructure resilience with application to electric power outages," Journal of Infrastructure Systems, vol. 19, no. 1, pp. 25-35, 2012.
[22] M. Ungar, "Qualitative contributions to resilience research," Qualitative Social Work: Research and Practice, vol. 2, no. 1, pp. 85-102, 2003.

[23] S. Sarre, C. Redlich, A. Tinker, E. Sadler, A. Bhalla, and C. McKevitt, "A systematic review of qualitative studies on adjusting after stroke: lessons for the study of resilience," Disability and Rehabilitation, vol. 36, no. 9, pp. 716-726, 2014.

[24] H. Baroud, K. Barker, and F. Hank Grant, "Multiobjective stochastic inoperability decision tree for infrastructure preparedness," Journal of Infrastructure Systems, vol. 20, no. 2, Article ID 04013012, 2013.

[25] L. Wang, X. Xue, R. J. Yang, X. Luo, and H. Zhao, "Built environment and management: exploring grand challenges and management issues in built environment," Frontiers of Engineering Management, vol. 6, no. 3, pp. 313-326, 2019.

[26] A. Cox, F. Prager, and A. Rose, "Transportation security and the role of resilience: a foundation for operational metrics," Transport Policy, vol. 18, no. 2, pp. 307-317, 2011.

[27] S. Enjalbert, F. Vanderhaegen, M. Pichon, K. A. Ouedraogo, and P. Millot, "Assessment of transportation system resilience," Human Modelling in Assisted Transportation, Springer, Berlin, Germany, pp. 335-341, 2011.

[28] O. Woolley-Meza, C. Thiemann, D. Grady et al., "Complexity in human transportation networks: a comparative analysis of worldwide air transportation and global cargo-ship movements," The European Physical Journal B, vol. 84, no. 4, pp. 589-600, 2011.

[29] A. Osei-Asamoah and N. E. Lownes, "Complex network method of evaluating resilience in surface transportation networks," Transportation Research Record: Journal of the Transportation Research Board, vol. 2467, no. 1, pp. 120-128, 2014.

[30] R. Pant, K. Barker, J. E. Ramirez-Marquez, and C. M. Rocco, "Stochastic measures of resilience and their application to container terminals," Computers \& Industrial Engineering, vol. 70, pp. 183-194, 2014.

[31] E. D. Vugrin, M. A. Turnquist, and N. J. K. Brown, "Optimal recovery sequencing for enhanced resilience and service restoration in transportation networks," International Journal of Critical Infrastructures, vol. 10, no. 3-4, pp. 218-246, 2014.

[32] M. Ouyang, L. Zhao, L. Hong, and Z. Pan, "Comparisons of complex network based models and real train flow model to analyze Chinese railway vulnerability," Reliability Engineering \& System Safety, vol. 123, pp. 38-46, 2014.

[33] R. Faturechi, E. Levenberg, and E. Miller-Hooks, "Evaluating and optimizing resilience of airport pavement networks," Computers \& Operations Research, vol. 43, pp. 335-348, 2014.

[34] R. Faturechi and E. Miller-Hooks, "Travel time resilience of roadway networks under disaster," Transportation Research Part B: Methodological, vol. 70, pp. 47-64, 2014.

[35] H. Baroud, K. Barker, J. E. Ramirez-Marquez, and C. M. Rocco, "Importance measures for inland waterway network resilience," Transportation Research Part E: Logistics and Transportation Review, vol. 62, pp. 55-67, 2014.

[36] D. Wang and W. H. Ip, "Evaluation and analysis of resilience and frangibility for transportation networks," Control Theory and Applications, vol. 27, no. 7, pp. 849-854, 2010, in Chinese.

[37] D. R. Wuellner, S. Roy, and R. M. D’Souza, "Resilience and rewiring of the passenger airline networks in the United States," Physical Review E, vol. 82, no. 5, Article ID 056101, 2010.

[38] L. Chen and E. Miller-Hooks, "Resilience: an indicator of recovery capability in intermodal freight transport," Transportation Science, vol. 46, no. 1, pp. 109-123, 2012. 
[39] W. H. Ip and D. Wang, "Resilience and friability of transportation networks: evaluation, analysis and optimization," IEEE Systems Journal, vol. 5, no. 2, pp. 189-198, 2011.

[40] E. Miller-Hooks, X. Zhang, and R. Faturechi, "Measuring and maximizing resilience of freight transportation networks," Computers \& Operations Research, vol. 39, no. 7, pp. 16331643, 2012.

[41] A. Cardillo, M. Zanin, J. Gómez-Gardeñes, M. Romance, A. J. García del Amo, and S. Boccaletti, "Modeling the multilayer nature of the European Air Transport Network: resilience and passengers re-scheduling under random failures," The European Physical Journal Special Topics, vol. 215, no. 1, pp. 23-33, 2013.

[42] K. Adjetey-Bahun, B. Birregah, E. Châtelet, J. L. Planchet, and E. Laurens-Fonseca, "A simulation-based approach to quantifying resilience indicators in a mass transportation system," in Proceedings of the 11th International ISCRAM Conference, pp. 75-79, University Park, PA, USA, May 2014.

[43] U. Bhatia, D. Kumar, E. Kodra, and A. R. Ganguly, "Network science based quantification of resilience demonstrated on the Indian Railways Network," PLoS One, vol. 10, no. 11, Article ID e0141890, 2015.

[44] M. Bruneau, S. E. Chang, R. T. Eguchi et al., "A framework to quantitatively assess and enhance the seismic resilience of communities," Earthquake Spectra, vol. 19, no. 4, pp. 733-752, 2003.

[45] J. C. Kelly, T. Ersal, C. T. Li et al., "Sustainability, resiliency, and grid stability of the coupled electricity and transportation infrastructures: case for an integrated analysis," Journal of Infrastructure Systems, vol. 21, no. 4, Article ID 04015001, 2015.

[46] M. Pregnolato, A. Ford, V. Glenis, S. Wilkinson, and R. Dawson, "Impact of climate change on disruption to urban transport networks from pluvial flooding," Journal of Infrastructure Systems, vol. 23, no. 4, Article ID 04017015, 2017.

[47] X. Xue, L. Wang, and R. J. Yang, "Exploring the science of resilience: critical review and bibliometric analysis," Natural Hazards, vol. 90, no. 1, pp. 477-510, 2018.

[48] M. E. Newman, S. H. Strogatz, and D. J. Watts, "Random graphs with arbitrary degree distributions and their applications," Physical Review E, vol. 64, no. 2, Article ID 026118, 2001.

[49] M. E. Newman, "Spread of epidemic disease on networks," Physical Review E, vol. 66, no. 1, Article ID 016128, 2002.

[50] R. Cohen, K. Erez, D. Ben-Avraham, and S. Havlin, "Resilience of the internet to random breakdowns," Physical Review Letters, vol. 85, no. 21, pp. 4626-4628, 2000.

[51] J. Shao, S. V. Buldyrev, R. Cohen, M. Kitsak, S. Havlin, and H. E. Stanley, "Fractal boundaries of complex networks," EPL (Europhysics Letters), vol. 84, no. 4, p. 48004, 2008.

[52] G. Galvan and J. Agarwal, "Community detection in action: identification of critical elements in infrastructure networks," Journal of Infrastructure Systems, vol. 24, no. 1, Article ID 04017046, 2017.

[53] S. V. Buldyrev, R. Parshani, G. Paul, H. E. Stanley, and S. Havlin, "Catastrophic cascade of failures in interdependent networks," Nature, vol. 464, no. 7291, pp. 1025-1028, 2010.

[54] L. Chang, F. Peng, Y. Ouyang, A. S. Elnashai, and B. F. Spencer Jr., "Bridge seismic retrofit program planning to maximize postearthquake transportation network capacity," Journal of Infrastructure Systems, vol. 18, no. 2, pp. 75-88, 2012.

[55] J. Johansson and H. Hassel, "An approach for modelling interdependent infrastructures in the context of vulnerability analysis," Reliability Engineering \& System Safety, vol. 95, no. 12, pp. 1335-1344, 2010.

[56] M. Ouyang, L. Hong, Z.-J. Mao, M.-H. Yu, and F. Qi, “A methodological approach to analyze vulnerability of interdependent infrastructures," Simulation Modelling Practice and Theory, vol. 17, no. 5, pp. 817-828, 2009.

[57] J. C. Chu and S.-C. Chen, "Optimization of transportationinfrastructure-system protection considering weighted connectivity reliability," Journal of Infrastructure Systems, vol. 22, no. 1, Article ID 04015008, 2015.

[58] L. Dueñas-Osorio, J. I. Craig, and B. J. Goodno, "Seismic response of critical interdependent networks," Earthquake Engineering \& Structural Dynamics, vol. 36, no. 2, pp. 285306, 2007.

[59] L. Dueñas-Osorio, J. I. Craig, B. J. Goodno, and A. Bostrom, "Interdependent response of networked systems," Journal of Infrastructure Systems, vol. 13, no. 3, pp. 185-194, 2007.

[60] Resilience Alliance, Assessing and Managing Resilience in Social-Ecological Systems: A Practitioners Workbook, Resilience Alliance, Stockholm, Sweden, 2007, http://library. uniteddiversity.coop/Transition_Relocalisation_Resilience/ resilience_practitioner_workbook_1.0.pdf.

[61] R. Zimmerman, Q. Zhu, F. de Leon, and Z. Guo, "Conceptual modeling framework to integrate resilient and interdependent infrastructure in extreme weather," Journal of Infrastructure Systems, vol. 23, no. 4, Article ID 04017034, 2017.

[62] L. Wang, X. Xue, Y. Zhang, and X. Luo, "Exploring the emerging evolution trends of urban resilience research by scientometric analysis," International Journal of Environmental Research and Public Health, vol. 15, no. 10, p. 2181, 2018.

[63] R. Albert, H. Jeong, and A.-L. Barabási, "Error and attack tolerance of complex networks," Nature, vol. 406, no. 6794, pp. 378-382, 2000.

[64] W. Najjar and J.-L. Gaudiot, "Network resilience: a measure of network fault tolerance," IEEE Transactions on Computers, vol. 39, no. 2, pp. 174-181, 1990.

[65] D. S. Callaway, M. E. J. Newman, S. H. Strogatz, and D. J. Watts, "Network robustness and fragility: percolation on random graphs," Physical Review Letters, vol. 85, no. 25, pp. 5468-5471, 2000.

[66] N. Eagle, M. Macy, and R. Claxton, "Network diversity and economic development," Science, vol. 328, no. 5981, pp. 1029-1031, 2010.

[67] C. E. Shannon, "A mathematical theory of communication," Bell System Technical Journal, vol. 27, no. 3, pp. 379-423, 1948.

[68] Department of Urban Surveys, National Bureau of Statistics of China, China City Statistical Yearbook-2015, China Statistics Press, Beijing, China, 2015.

[69] China Railway Customer Service Center, 2015, http://www. 12306.cn/.

[70] Civil Aviation Administration of China, 2015, http://www. arnet.gov.

[71] The Open Graph Viz Platform, 2015, http://www.https:// gephi.org/.

[72] M. Newman, Networks: An Introduction, Oxford University Press, Oxford, UK, 2010.

[73] D. Matyas and M. Pelling, "Positioning resilience for 2015: the role of resistance, incremental adjustment and transformation in disaster risk management policy," Disasters, vol. 39, no. 1, pp. s1-s18, 2015.

[74] S. Meerow, J. P. Newell, and M. Stults, "Defining urban resilience: a review," Landscape and Urban Planning, vol. 147, pp. 38-49, 2016. 
[75] M. Barthélemy, "Spatial networks," Physics Reports, vol. 499, no. 1-3, pp. 1-101, 2011.

[76] J. Gao, B. Barzel, and A.-L. Barabási, "Universal resilience patterns in complex networks," Nature, vol. 530, no. 7590, pp. 307-312, 2016. 